Questions de communication la politique

\title{
Classer, marchandiser et manager : quel idéal de l'Université opposer aux dérives en cours?
}

Classify, Commodify and Manage: What Ideals of the University oppose to the Ongoing Excesses?

\section{Arnaud Mercier}

\section{OpenEdition}

\section{Journals}

Édition électronique

URL : http://journals.openedition.org/questionsdecommunication/8675

DOI : 10.4000/questionsdecommunication. 8675

ISSN : 2259-8901

\section{Éditeur}

Presses universitaires de Lorraine

\section{Édition imprimée}

Date de publication : 31 décembre 2013

Pagination : 125-144

ISBN : 978-2-8143-0182-5

ISSN : 1633-5961

\section{Référence électronique}

Arnaud Mercier, «Classer, marchandiser et manager : quel idéal de l'Université opposer aux dérives en cours? ", Questions de communication [En ligne], 24 | 2013, mis en ligne le 01 février 2016, consulté le 12 mai 2019. URL : http://journals.openedition.org/questionsdecommunication/8675 ; DOI : 10.4000/ questionsdecommunication.8675 


\title{
$>$ ÉCHANGES
}

ARNAUD MERCIER

Centre de recherche sur les médiations

Université de Lorraine

F-57000

arnaud.mercier@univ-lorraine.fr

\section{CLASSER, MARCHANDISER ET MANAGER : QUEL IDÉAL DE L'UNIVERSITÉ OPPOSER AUX DÉRIVES EN COURS?}

\begin{abstract}
Résumé. - Pour poursuivre le débat sur les dérives des universités, nous revenons sur les objections formulées par les contributeurs de la rubrique «Échanges 》. Cela permet de mieux souligner les perversités à l'œuvre dans les usages qui sont faits du fameux « classement de Shanghai » des universités. C'est aussi l'occasion de souligner les dérives financières, causées par une tendance lourde au désengagement des États. En Amérique du Nord, l'endettement des étudiants prend des allures de bulle spéculative qui rappelle dangereusement les excès des tristement célèbres subprimes. Enfin, le texte propose une modeste réflexion sur le type de réponse collective que les universitaires peuvent fournir face aux dérives constatées.
\end{abstract}

Mots clés. - Universités, endettement des étudiants, classement de Shanghai, LRU, fusion des universités. 
Le texte d'Arnaud Mercier (2012) a donné lieu à des «Échanges » sur le thème « Dérives des universités, périls des universirtaires ». II revient à son auteur de répondre aux chercheurs qui ont discuté ses propositions.

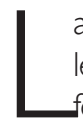
a qualité critique des textes écrits en réaction à notre article (Mercier, 20I2) sur le destin des universités, hébergé dans cette rubrique « Échanges 》, prouve une fois encore que l'enrichissement intellectuel naît de la confrontation des points de vue. Remercions donc les collègues qui ont pris le temps de nous lire et d'alimenter le débat sur la façon d'exercer le métier d'enseignant-chercheur à l'avenir. Au-delà de ce que chacun affirme partager comme similarité dans les constats et analyses proposés, beaucoup ont choisi de développer leur argumentaire en complémentarité avec notre contribution liminaire. Mais, les uns et les autres pointent aussi au passage des désaccords sur l'analyse ou sur certaines idées émises. Nous voudrions donc, à notre tour, mettre en exergue des points d'accord avec certains compléments apportés et prolonger la réflexion, tout en reformulant et défendant parfois plusieurs de nos arguments face aux critiques énoncées. Le propos sera structuré en trois temps : l'un consacré aux classements des universités et à leurs mésusages, un deuxième présentant les méfaits de la marchandisation du savoir sur l'endettement des étudiants en Amérique du Nord, un dernier centré sur les conditions d'engagement des universitaires contre les transformations en cours de l'Université.

\section{Les classements académiques et leurs mésusages: la course erronée au gigantisme}

Julie Bouchard (20/3) apporte un éclairage enrichissant sur les enjeux de gouvernance et médiatiques qui entourent la publicisation des résultats des classements internationaux des universités. Les recherches qu'elle a conduites, avec d'autres collègues, sur ce thème donnent un éclairage plus édifiant encore que ce nous percevions comme un problème, en simple qualité « d'usager » des universités. Ses analyses sur les usages autopromotionnels - par les universités elles-mêmes - de ces classements exogènes aux critères souvent assez arbitraires, font froid dans le dos. Pour les universités qui s'en vantent, c'est prendre le risque de se tirer une balle dans le pied! Car les critères ouvrent grand la porte à des aléas de classement, alors qu'ils assurent la stabilité des universités classées en tête, puisque les critères sont issus de l'observation de leur situation et principes de fonctionnement.

II faut rappeler inlassablement que le « célèbre » classement dit de Shanghai repose sur un modèle d'université à l'américaine et que le système de structuration de l'enseignement supérieur et de la recherche en France le fait d'emblée partir avec un handicap. Enfin, les gouvernants français le disent comme nous le verrons! Puisque nous avons un grand organisme public de recherche pluridisciplinaire (le CNRS - Centre national de la recherche scientifique) plus d'autres entités comme l'Inserm (Institut national de la santé et de la recherche médicale) pour la santé ou I'lfremer (Institut français de recherche pour l'exploitation de la mer) 
pour les océans, puisqu'une partie de la formation des élites est assurée par des « grandes écoles » ou des « grands établissements publics d'enseignement supérieur », avec des étudiants provenant de filières sélectives dissociées de l'Université (les classes préparatoires), le système universitaire français dans son ensemble n'est simplement pas comparable à l'aune des critères retenus.

Redisons donc avec force que si les universités françaises commencent à agir principalement en fonction de ces classements, elles prennent le risque d'y perdre une partie de leur âme. Un cas exemplaire de cette dérive liée à l'obsession du classement est la course au gigantisme. On pourrait dire beaucoup de choses sur notre propre université fusionnée (celle de Lorraine), mais, afin de ne pas essuyer le reproche de régler des comptes, nous préférons évoquer le cas des trois ex-universités d'Aix-Marseille. Exposons d'abord quelques extraits du texte de présentation de la nouvelle entité fusionnéel :

«Aix-Marseille Université [AMU] a été créée par le décret n 20 I I- I 010 du 24 août 20 I I. Elle est entrée en vigueur le ler janvier 20 I2, se substituant à l'université de Provence, de la Méditerranée et Paul-Cézanne. C'est aujourd'hui une des plus jeunes universités de France, c'est aussi la plus grande de par le nombre de ses étudiants, de ses personnels et par son budget. C'est également la plus grande université francophone. Autant d'atouts, au-delà des résultats d'ores et déjà notables dans le domaine de l'enseignement et de la recherche, qui font d'Aix-Marseille Université un établissement d'enseignement supérieur et de recherche d'excellence [...].

\section{Une reconnaissance internationale d'ores et déjà indéniable}

Lors du dernier classement international de Shanghai, paru le 15 août 20II, Aix-Marseille Université s'est positionnée dans la tranche 102-150 du classement mondial sur 500 universités classées (c'est un progrès notable par rapport au classement des universités évaluées séparément) et dans la tranche 4-6 des universités françaises ».

On voit ici que le gigantisme est posé en atout incontestable et qu'il a valeur en soi de « reconnaissance » accrue dans les classements internationaux, dont il convient de se vanter. Mais lorsque le verdict 2013 tombe mi-août, et que l'Amu recule à la $15 l^{\mathrm{e}}$ place, son président Yvon Berland use sur le site, de circonlocutions pour préciser qu'une involution n'est en revanche pas si grave - «Quelques places simplement ont été perdues $\gg$ - puisque, si l'AMU a changé de tranche ( I I -200), cela ne représente que six place de moins, car lorsqu'elle se vante sur son site d'être dans la tranche 102-150, ce marketing grossier cache qu'elle était en fait en fin de peloton (à la $145^{\mathrm{e}}$ place). C'est un même désagrément que subit l'université de Lorraine dont la fusion (des trois universités de Nancy et de celle de Metz) devait assurer une montée en puissance dans le classement de Shanghai et qui fait du surplace en légère régression : en 2011 , 273e place ; en 2012, 277e ; en 2013 :278e.

La course au gigantisme est encouragée depuis plus de dix ans par les autorités politiques françaises. Un rapport sénatorial (co-rédigé par deux élus, l'une socialiste, l'autre de I'Union du mouvement populaire - UMP) sur le bilan de la Loi relative aux libertés et responsabilités des universités (la fameuse LRU) et du dispositif de

\footnotetext{
' Accès : http://www.univ-amu.fr/actualites/presentation-luniversite. Consulté le 20/08//3.
} 
rapprochement des universités mettant en commun certaines tâches (dispositifs appelés pôles de recherche et d'enseignement supérieur - PRES) publié en mars 2013, en vient à regretter le manque d'audace de beaucoup d'universités en la matière, même si le mouvement est lancé et irait dans le bon sens car les universités françaises deviendraient enfin assez grosses pour être visibles dans les classements :

« Ces regroupements universitaires ont été constitués dans une logique de seuil, par la mutualisation tant des capacités de recherche et de formation que des publics étudiants accueillis. Ils ont permis d'établir des périmètres universitaires plus comparables aux grands ensembles universitaires prestigieux occupant une place de choix dans les comparaisons internationales. À cet égard, il est intéressant de rappeler que l'université de Bologne accueille pas moins de 85000 étudiants répartis sur de multiples sites, soit deux fois plus qu'une "grande" université française (l'université de Strasbourg compte, après la fusion de ses entités constitutives, 43000 étudiants) » (Gillot, Dupont, 20 I3).

Cet argument est faux et pire, il est aussi erroné qu'absurde. Le lecteur informé en est réduit aux conjectures pour comprendre comment un tel mensonge est si complaisamment relayé : les politiques sont-ils incompétents sur ce sujet (hélas, on ne peut exclure l'hypothèse quand on voit que le Sénat avait voté en catimini, sans débat, la suppression de la procédure de qualification aux emplois d'enseignants-chercheurs par le Conseil national des universités (CNU), au printemps) ? Sont-ils manipulateurs et osent-ils, du coup, affirmer une contre-vérité en suivant l'adage « plus c'est gros mieux ça passe »? Ou sont-ils victimes d'une auto-intoxication faisant prendre les représentations culturelles qui traînent pour la vérité, au point de ne pas juger utile de vérifier les données. Apportons à ces deux sénateurs les données qui leur manquent, via ce tableau mettant en relation les douze premières universités du classement de Shanghai 2013 avec leur nombre approximatif d'étudiants.

Tableau I. Effectifs étudiants des universités en tête du classement de Shangai².

\begin{tabular}{|c|l|r|}
\hline Rang & Université & $\begin{array}{l}\text { Nombre } \\
\text { d'étudiants }\end{array}$ \\
\hline 1 & Harvard & 21000 \\
\hline 2 & Stanford & 15800 \\
\hline 3 & Berkeley & 35000 \\
\hline 4 & Massachusetts Institute of Technology (MIT) & 10900 \\
\hline 5 & Cambridge & 18500 \\
\hline 6 & California Institute ofTechnology & 2200 \\
\hline 7 & Princeton & 7600 \\
\hline 8 & Columbia & 28900 \\
\hline 9 & Chicago & 12300 \\
\hline 10 & Oxford & 21500 \\
\hline 11 & Yale & 11700 \\
\hline 12 & University of California & 40000 \\
\hline
\end{tabular}

2 Source pour les effectifs : wikipédia (consulté en août 2013), support qui, à défaut d'être absolument fiable, suffit à donner une indication du dimensionnement de ces universités. 
Nul besoin de décrypter les chiffres pour comprendre d'un seul coup d'œil que la visibilité dans le classement de Shanghai pour les universités leaders n'a rien à voir avec le nombre massif d'étudiants et la course au gigantisme qu'on impose en France, en la parant de toutes les vertus. Et, s'il faut sacrifier à l'air du temps comparatif et classificatoire, alors comparons les données essentielles des universités en tête du classement avec quelques universités françaises « bien classées » et/ou en proie au gigantisme par fusion. La mise en parallèle du nombre d'étudiants, d'enseignants-chercheurs et des budgets (par indulgence pour les universités françaises, nous avons omis d'intégrer les indispensables personnels administratifs et techniques). Le bilan est sans appel. Cinquième du classement de Shanghai 2013, l'université de Cambridge, fait figure de parent pauvre par rapport à ses rivales, avec un budget par étudiant de seulement 5 I 000 €, mais à égalité quand même avec Berkeley. En face, les mastodontes universitaires français fusionnés font pâle figure avec une moyenne de $10000 €$ par étudiant, loin derrière les $132000 €$ par étudiant de la tête de classe : l'université d'Harvard.

Tableau 2. Universités françaises fusionnées et celles en tête du classement de Shanghai : douloureuses comparaisons ${ }^{3}$.

\begin{tabular}{|c|c|c|c|}
\hline Universités & $\begin{array}{l}\text { Nombre } \\
\text { d'étudiants }\end{array}$ & $\begin{array}{l}\text { Ratio étudiants par } \\
\text { enseignant-chercheur }\end{array}$ & $\begin{array}{l}\text { Ratio budget } \\
\text { par étudiant }\end{array}$ \\
\hline $\begin{array}{l}\text { Université d'Harvard } \\
\text { corps enseignant : } 2 \text { I00 personnes } \\
\text { Budget } 2012: 3,7 \text { milliards } \$\end{array}$ & 21000 & 10 & $\begin{array}{r}176190 \$ \\
(132000 €)\end{array}$ \\
\hline $\begin{array}{l}\text { Université de Stanford } \\
\text { corps enseignant : I } 995 \text { personnes } \\
\text { Budget pour la recherche en } 2012: \text { 1,27 milliards } \$\end{array}$ & 15800 & 8 & $\begin{array}{r}80300 \$ \\
(60200 €)\end{array}$ \\
\hline $\begin{array}{l}\text { Université de Berkeley } \\
\text { | } 582 \text { enseignants temps plein }+500 \text { à temps partiel } \\
\text { Budget en } 20 \text { । } 0 \text {-20 I I : } 2,4 \text { milliards } \$\end{array}$ & 35000 & 16,8 & $\begin{array}{r}68500 \$ \\
(51300 €)\end{array}$ \\
\hline $\begin{array}{l}\text { Massachusetts Institute of Technology (MIT) } \\
\text { I } 753 \text { professeurs (tous rangs confondus) } \\
\text { + autres personnels enseignants } \\
\text { Budget en } 2012: 2,7 \text { milliards } \$\end{array}$ & 10900 & 6,2 & $\begin{array}{r}247000 \$ \\
(185000 €)\end{array}$ \\
\hline $\begin{array}{l}\text { Université de Cambridge } \\
2845 \text { membres rattachés à la recherche } \\
\text { Budget en } 2012: 810 \text { millions } t\end{array}$ & 18500 & 6,5 & $\begin{array}{r}43800 € \\
(51300 €)\end{array}$ \\
\hline $\begin{array}{l}\text { Université Paris-Sud } \\
2460 \text { enseignants-chercheurs } \\
\text { Budget en } 2012: 400 \text { millions } €\end{array}$ & 27000 & 11 & $14800 €$ \\
\hline $\begin{array}{l}\text { Université de Lorraine } \\
3700 \text { enseignants-chercheurs Budget en 2013: } \\
562 \text { millions } €\end{array}$ & 52400 & 14 & $10700 €$ \\
\hline $\begin{array}{l}\text { Université de Strasbourg } \\
2780 \text { enseignants-chercheurs } \\
\text { Budget en } 2012: 443 \text { millions } €\end{array}$ & 43000 & 15,6 & $10300 €$ \\
\hline $\begin{array}{l}\text { Aix-Marseille Université } \\
4600 \text { enseignants-chercheurs } \\
\text { Budget } 2012: 650 \text { millions } €\end{array}$ & 72000 & 15,6 & $9000 €$ \\
\hline
\end{tabular}

\footnotetext{
3 Sources : sites de chacune des universités (consultés en août 2013).
} 
II faut donc d'urgence réclamer aux décideurs politiques de QUINTUPLER LES BUDGETS des universités françaises pour faire des bonds importants dans le classement de Shanghai! Et nous attendons avec impatience qu'un décideur universitaire ou politique ose dire que la demande est absurde car ce tableau n'a pas de sens, dès lors que l'on compare des budgets issus de traditions et de modes de financement si différents. Nous serons alors encore plus fondé à leur renvoyer en boomerang que légitimer le gigantisme pour grimper dans le classement de Shanghai est tout aussi absurde, pour les mêmes raisons d'univers incomparables a priori.

L'intérêt du texte de Julie Bouchard (20 I 3) tient aussi au rappel que le problème de ces classements académiques tient largement aux usages qui en sont fait : par les universités elles-mêmes ou par les acteurs politiques nationaux, régionaux et locaux, pas toujours versés aux subtilités des problèmes méthodologiques de leur élaboration, pour qui ces classements peuvent apparaître plus objectifs qu'ils ne le sont. Mais, le phénomène le plus pervers est celui décrit par l'auteure qui fait que la contestation des principes du classement contribue, en réalité, à renforcer l'idée du classement en soi : « Ce type d'opposition aux classements partage avec les promoteurs de classements existants l'idée selon laquelle il existerait un classement susceptible de bien ou mieux refléter le réel » (Bouchard, 2013: |9|). Et l'on voit que des politiques peuvent intégrer la critique des principes du classement de Shanghai, tout en faisant comme si cela n'était pas si grave et qu'il fallait quand même se réjouir d'y figurer et d'y progresser. Le ministre de l'Enseignement supérieur et de la Recherche, Geneviève Fioraso, déclara sur France Info (citée par Reuters, |5/08/I3) : « II faut reconnaître les limites du classement de Shanghai qui a été modelé sur un modèle anglo-saxon mais en même temps la France, petit à petit, globalement, grignote des places dans le classement donc c'est plutôt pas mal ».

Pour autant, un point de divergence apparaît avec Julie Bouchard. Critiquer les principes méthodologiques n'est pas forcément participer à la célébration de ces classements, si les attaques visent à écorner leur légitimité même. Et, précisément, les citations que la chercheuse a retenues de notre texte visent à saper leur légitimité et non à les améliorer au nom d'un idéal de classement qui serait meilleur : «Ce classement ne mesure pas la performance de nos établissements mais leur écart à la norme américaine qui a servi à en dicter les critères. Vouloir nous faire croire qu'à coup de meilleures performances ou de fusions, nous entrerons aux meilleures places de ce classement n'est rien d'autre qu'une absurdité » (Mercier, 2012 : 209). Ce disant, notre analyse ne manifeste à l'évidence aucune valeur partagée avec les promoteurs de ce classement. En ce sens, il apparaît que qualifier ces classements de « miroir déformant » n'est en rien incompatible avec la riche conceptualisation de la notion de « classement » proposée par Julie Bouchard (2013), contrairement à ce qu'elle laisse entendre. 


\section{La marchandisation de l'enseignement supérieur et le périlleux endettement des étudiants : le syndrome nord-américain}

Parmi les apports des articles d'Éric George (2013) et de Julien Duval (2013), retenons la dimension économique qui fut, en effet, trop absente de notre démonstration. Économiste de son état, le second auteur pointe avec beaucoup d'acuité le développement chez les décideurs politiques et académiques d'une vision étroite de l'utilitarisme qui sert un schéma d'économisation de l'Université. Travaillant dans les universités canadiennes, le premier met fort bien en exergue une autre dérive, celle des droits d'inscription et de l'endettement des étudiants, singulièrement aux États-Unis - qui est très proche d'une bulle spéculative - mais aussi au Canada.

\footnotetext{
« Ces questions prennent une coloration spécifique dans un contexte d'endettement croissant des étudiants. Ceux-ci apparaissent de plus en plus concernés par des questions matérielles de subsistance : ce qui entraîne soit des emplois du temps particulièrement chargés entre travail, voire petits boulots, et université, soit un endettement considérable qui pèse ensuite pendant plusieurs années ; à moins que les deux soient combinés. On peut comprendre pourquoi, de leur point de vue, l'Université a avant tout pour but de les aider à obtenir un emploi sur le marché du travail capitaliste » (George, 2013 : 245).
}

Et la démonstration conduite par notre collègue apporte une précieuse mise en évidence d'un cercle vicieux : la marchandisation de l'Université, l'orientation vers ce qui devrait devenir sa finalité première, voire unique (la professionnalisation) s'auto-entretient, faisant des étudiants des clients qui estiment à bon droit devoir obtenir des résultats tangibles et avérés, bénéficier d'une individualisation de leur parcours, d'autant plus qu'ils paient cher. La logique néomanagériale appliquée dans I'Université tend donc à « exacerber la tendance à l'utilitarisme de la part des étudiants, à la focalisation sur les résultats, sur l'obtention du titre au détriment d'une réelle implication dans le processus d'acquisition des savoirs » (George, 2013 : 245).

Revenons donc sur la situation d'endettement des étudiants américains pour voir jusqu'à quelles dérives l'introduction de pratiques ultralibérales peut conduire, à force de tailler dans les subventions publiques aux universités, à les inciter à accroître leurs frais d'inscription, voire à entrer en compétition pour capter des étudiants, leur vendant fort cher un rêve de « diplomation » et d'emploi.

Au cours des trois dernières décennies, les coûts d'inscription - jusqu'à l'obtention d'un diplôme de bachelor (équivalent à celui de la licence dans le système licence-master-doctorat - LMD) - ont augmenté de plus de I 000 \% (Johnson, Van Ostern, White, 2012 : I). En 201I, deux tiers des étudiants qui obtiennent le bachelor en quatre ans l'ont avec une dette moyenne d'un peu plus de 25000 \$. Le total de l'endettement étudiant a atteint la somme 
astronomique de plus de 920 milliards de dollars, et avec d'autres estimations cela dépasse même déjà les I 000 milliards. «L'une des principales causes est la diminution constante du financement de l'État pour l'enseignement supérieur, qui avait aidé les colleges à conserver des frais de scolarité abordables. Les coûts d'inscription dans les colleges et universités croissant régulièrement et rapidement, sur tout le territoire, cela a provoqué une hausse spectaculaire de l'endettement des étudiants $\gg^{4}$ (ibid.). Ce taux d'endettement est en hausse constante depuis plus de dix ans et se répartit avec l'effet du temps et de l'aide des parents à leurs enfants, sur l'ensemble des générations comme le montre le graphe issu d'une étude publiée en mars 2013 de la Federal Reserve Bank of New York (FRBNY - Lee, $2013: 4)^{5}$.

Tableau 3. Répartition de l'endettement global étudiant par tranches d'âge (source : FRBNY Consumer Credit Panel).

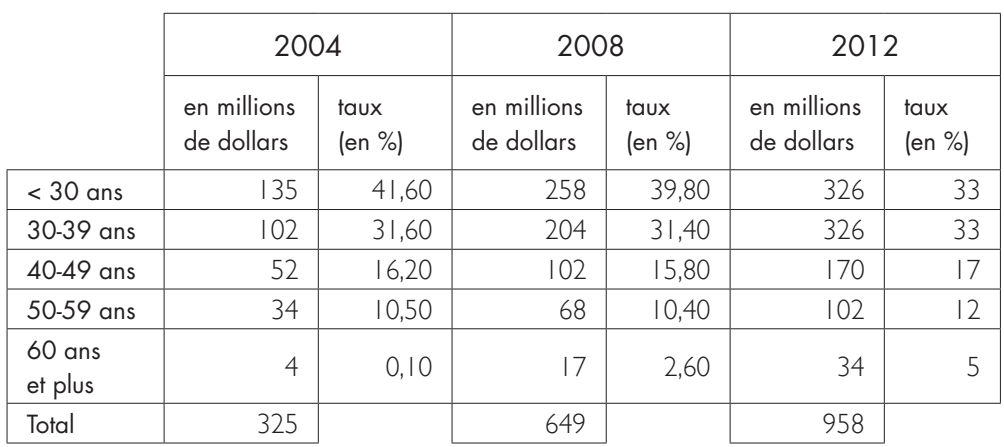

La dette moyenne par étudiant emprunteur est de 24000 \$, mais cette moyenne est peu significative, car certains ont accumulé des passifs bien plus lourds. Le camembert de répartition proposé par la FRBNY est plus parlant (Lee, 2013 : 6). Ainsi découvre-t-on que $40 \%$ ont moins de $10000 \$$ de dettes (7 $500 €$ ), que $30 \%$ ont entre 10000 et 25000 (18 $700 €$, ce qui obère déjà un peu les capacités à vivre bien ses premières années dans la vie active), mais cela signifie donc que $30 \%$ ont plus à rembourser, $27 \%$ devant entre 25000 et $100000 \$$ (75 $000 €$ !). Une pareille somme place les diplômés en situation de risque élevé : il leur faudra absolument trouver du travail, voire en avoir deux ou trois pour cumuler les revenus ; l'accès à d'autres crédits (consommation, logement, santé...) va devenir très difficile au vu du taux d'endettement déjà atteint (ce que remarque la FRBNY dans son étude, s'inquiétant du tarissement à venir de la consommation pour ces nouvelles générations, dont une part importante du revenu servira d'abord à rembourser ces crédits).

\footnotetext{
${ }^{4}$ Nous traduisons.

${ }^{5}$ Accès:http://wnww.newyorkfed.org/newsevents/mediaadvisory/20 I 3/Lee0228 I 3.pdf. Consulté le 23/08/I3.
} 
Tableau 4. Distribution de la dette étudiante par paliers financiers (source : FRBNY Consumer Credit Panel).

\begin{tabular}{|r|r|}
\hline Somme de la dette (en \$) & $\begin{array}{l}\text { Taux } \\
\text { d'étudiants } \\
\text { (en \%) }\end{array}$ \\
\hline 1 I à 10000 & 40 \\
\hline 10000 à 25000 & 29,70 \\
\hline 25000 à 50.000 & 17,70 \\
\hline 50000 à 100.000 & 9 \\
\hline 100000 à I 50.000 & 2,20 \\
\hline 150000 à 200.000 & 0,90 \\
\hline$>200000$ & 0,60 \\
\hline Total & 100,00 \\
\hline
\end{tabular}

Selon cette étude, les raisons de la croissance de la dette par personne et des emprunteurs sont multiples :

- plus de gens fréquentent les collèges et font des études supérieures ;

- plus de parents inquiets pour l'avenir de leurs enfants prennent des prêts étudiants pour leur permettre de faire des études qu'ils ne suivraient pas sans cette aide familiale ;

- les étudiants qui suivent des études supérieures restent plus longtemps à l'Université pensant accroître ainsi leurs chances de trouver du travail à la sortie ;

- la baisse des taux de remboursement par les emprunteurs avec la montée des retards de paiements ou des reports, accroît le montant des crédits dus. D'autant que, en 2005, les sénateurs républicains ont inscrit dans une loi l'exclusion des crédits pour étude de la liste des crédits entrant dans la procédure de surendettement et pouvant aboutir à déclarer l'état de faillite personnelle.

En effet, il faut relier cette question aux perspectives d'emploi, comme le fait le Project on Student Debt at The Institute for College Access \& Success (TICAS), dans une note de $2012^{6}$ mettant en perspective les faits sur plusieurs années. Le taux de chômage des jeunes diplômés était de 8,8 \% en 20। I, soit une légère baisse par rapport au record de 2010 de $9,1 \%$. Le constat est que beaucoup plus de jeunes diplômés sont sous-employés, travaillant seulement à temps partiel ou occupant des emplois moins bien rémunérés ne nécessitant pas une formation supérieure. Les étudiants qui ont emprunté pour étudier et qui ont obtenu un bachelor en 20 II, l'ont fait avec en moyenne $26600 \$$ de dette de prêt étudiant, contre 25250 \$ en 2010, 23280 \$ en 2008 et $17000 \$$ en 1996. L'augmentation de 2010-201। de $5 \%$ est similaire à celle annuelle moyenne au cours des dernières années selon le TICAS. Le rapport

\footnotetext{
${ }^{6}$ Accès : http://www.ticas.org/files/pub//Release_SDRI2_I0 |8|2.pdf. Consulté le 13/| |/|3.
} 
(The Institute for College Access \& Success, 2012) a également constaté qu'environ deux tiers des étudiants inscrits en 201 I avaient des prêts pour étude, chiffre en croissance constante.

On comprend mieux pourquoi le désengagement de la dette après les études devient très difficile et que le solde qui reste aux emprunteurs diplômés s'accroisse alors que le nombre de ceux qui suivent leurs études endettés croît aussi. Selon les données rassemblées par l'American Student Assistance', sur les 37 millions d'emprunteurs américains qui ont des soldes impayés de prêts étudiants, 14 \%, soit environ 5,4 millions d'emprunteurs, ont au moins une ligne de crédit étudiant (il est fréquent qu'ils empruntent à plusieurs organismes) totalement en souffrance et sont, dès lors, considérés comme « delinquents ». Et deux étudiants emprunteurs sur cinq - 4l \% - sont en défaillance de paiement à un moment donné, au cours des cinq premières années après le début du remboursement. Et, si tous les étudiants sont plus ou moins fragilisés, il est une catégorie qui devient sinistrée, ceux qui abandonnent leurs études avant le diplôme, alors qu'ils ont emprunté, parfois beaucoup. Selon la chercheuse Mary Nguyen (20 I 2), les tendances sont « inquiétantes » sur ce point :

- de 200 I à 2009, le taux d'étudiants qui ont emprunté pour financer leurs études en college est passé de $47 \%$ à $53 \%$;

- parmi ceux qui ont emprunté, le taux d'étudiants qui ont abandonné entre 2003 et 2009 était plus grand que celui de ceux qui avaient abandonné entre 1995 et 200I ;

- ceux qui ont abandonné ont des taux de chômage plus élevés et gagnent moins d'argent que ceux ayant obtenu leur diplôme. Les emprunteurs qui ont abandonné leurs études avant la fin sont quatre fois plus susceptibles de faire défaut sur leurs prêts ;

- les tendances sont plus marquées dans le secteur académique à but lucratif, qui a beaucoup grossi au cours des années 2000, en partie en raison d'un fort recrutement et de l'enrôlement des étudiants de familles à faible revenu en les y encourageant par une politique de crédit alléchante et agressive.

Le pire est que tout cela peut être le fruit d'un cercle vicieux très bien décrit ici, et que les syndicats d'étudiants en France dénoncent aussi souvent, même si, aux États-Unis, le phénomène est d'une toute autre ampleur et gravité.

« Les données montrent comment les coûts toujours croissants des College mettent de plus en plus d'étudiants entre le marteau et l'enclume : ce qu'ils doivent faire pour éviter d'emprunter trop d'argent, comme l'inscription à temps partiel et des emplois à temps plein en parallèle, rend également plus difficile pour eux l'obtention de leur diplôme. Même si le marché de l'enseignement supérieur devient plus fourni et complexe, les conséquences des mauvais choix sont de plus en plus sévères » ${ }^{8}$ (ibid. : | -2).

\footnotetext{
7 Accès : http://www.asa.org/policy/resources/stats/. Consulté le 25/08/l 3.

${ }^{8}$ Nous traduisons.
} 
Avec la baisse tendancielle des soutiens publics et la compétition accrue entre les établissements pour attirer les étudiants et accroître leurs ressources propres, la marchandisation à outrance de l'enseignement supérieur s'est faite « grâce » à des tactiques agressives des prêteurs que Richard Cordray, directeur du Bureau de protection financière des consommateurs, a qualifié de tactiques « étonnamment similaires » (《strikingly similar », cité in : Johnson, Ostern, White, 2012:7) à celles de l'industrie des prêts hypothécaires lorsque les funestes subprimes sont montés en flèche. Le résultat est une augmentation globale de la précarité financière des diplômés et un appauvrissement net des générations sorties sans diplôme avec la création d'une bulle spéculative qui semble ne pas avoir grand-chose à envier aux tristement célèbres subprimes. Selon « le gestionnaire de "fonds de couverture" (hedge-fund) Steve Eisman, s'exprimant devant le Congrès américain [...], l'industrie de l'éducation à but lucratif est tout aussi destructrice que l'industrie des hypothèques subprimes. Selon lui, nous assistons aux débuts d'une bulle semblable à la crise des hypothèques subprimes où les établissements, donc les recruteurs, qui sont payés à la commission, se battent pour recruter les étudiant e 's avant de les jeter à la rue bardés non pas de diplômes, mais de dettes financées par l'État »?

\section{Idéalisation de l'Université et capacité à (ré)agir collectivement}

Julien Duval (2013 :2 I2) souligne avec pertinence que nos descriptions du système universitaire tel qu'il est attaqué et miné de l'intérieur, relèvent parfois du « portrait idéalisé ». Julie Bouchard (2013) va dans le même sens. Cette forme d'idéalisation peut conduire sans doute, quelque fois, à sortir du cadre étroit de l'analyse froide et « objective », propre, dit-on, au jugement scientifique. En ce sens, nous n'avons pas détaillé les rapports de force au cœur du système universitaire, les différences entre les domaines de formation, les statuts, etc. Remercions donc Julien Duval (2013) ou Marc Lits et Évelyne Léonard (2013) de l'avoir fait pour nous. « On a affaire à une organisation qui présente une structuration apparemment forte (hiérarchies, grades, règlements, procédures et tutti quanti) mais qui laisse une large place à d'innombrables champs de force entre les acteurs qui la composent et ceux avec qui elle est en relation (politiques, institutions concurrentes, financeurs, etc.) » (ibid. : 270). Occupé à notre démonstration sur les évolutions (ne faudrait-il pas dire plutôt les involutions ?) de l'Université française, nous avons opté pour une approche idéaltypique à la Max Weber, rassemblant ce qui permet de définir des traits communs plutôt que d'entrer dans les détails de ce qui a toujours fait les ferments d'opposition interne à la communauté universitaire. Nous sommes donc bien d'accord pour affirmer qu'au-delà de ce qui unifie nos destins, l'Université s'apparente, comme l'a si bien décrit Christine Musselin (1997), à une « anarchie

\footnotetext{
9 Accès : http://www.iris-recherche.qc.ca/wp-content/uploads/20 I2/03/Note-Endettement-web.pdf Consulté le 23/08/13.
} 
organisée » et que c'est aussi dans ces conceptions divergentes qui ont toujours existé que les tenants du néomanagérialisme peuvent trouver des « alliés 》 en interne, pour appliquer à tous, des préceptes qu'une majorité du corps enseignant rejette pourtant. Car, comme le souligne avec force (véhémence ?) Éric Dacheux (20 I3), les réformes néomanagériales sont mises en œuvre par des universitaires, et nous sommes donc collectivement responsables de notre malheur, du moins en partie, nuancerions-nous quand même...

Cet exercice d'autoflagellation étant réalisé, il faut aussitôt affirmer que nous n'avançons pas honteux. Oui, il y a une part d'idéalisation dans l'opposition binaire proposée entre les divers éléments qui construisent deux conceptions différentes de l'Université. Ce qui n'interdit toutefois pas des combinaisons, des hybridations de modèles, avions-nous pris soin de préciser. Heureusement qu'il y a possibilité d'exprimer, même dans un article académique, une forme d'idéal, car, justement, l'un des défis auxquels les universitaires font face est de ne pas laisser les acteurs extérieurs leur imposer, sur le mode de l'évidence, leur propre idéalisation. C'est aussi sur la capacité à définir par nous-mêmes, pour nous-mêmes, notre idéal du métier, que nous sortirons d'une spirale dans laquelle l'application des principes imposés nous enferme. Nous nous sentons donc totalement en phase avec la conclusion de Marc Lits et Évelyne Leonard (2013 : 273) : « L'enjeu consiste donc à refonder un projet commun, dans un contexte où des forces centrifuges sont à l'œuvre, en interne, et des concurrences dures sont en confrontation, dans l'espace national comme devant l'internationalisation croissante ». Notre article se voulait une contribution à la fois à un diagnostic précis et chirurgical et à une définition d'une certaine idée de l'Université.

Car les travaux de sociologie des mobilisations enseignent que la capacité à agir et mobiliser repose notamment sur l'aptitude à « construire sa cause », en créant et propageant un idéal à atteindre (des utopies) ou à défendre (des acquis), reposant souvent sur « l'invention d'une tradition » (Hobsbawm, Ranger, 1983), la définition de « mythologies politiques » (Girardet, 1986), la manipulation de symboles et « d'émotions politiques » (Braud, 1996), de « passions politiques » (Ansart, 1983). D'ailleurs, Éric Dacheux (2013 : 203) défend la même idée au sujet de « la tension - nécessaire selon Paul Ricœur (1997) - entre utopie et idéologie » qui a, selon lui, « déserté l'université.Aujourd'hui, 1968 n'est plus qu'un lointain souvenir, le peu de débat contradictoire sur l'intérêt général que connaît notre communauté ne permet pas l'élaboration d'une utopie universitaire qui, à l'image du projet porté par le collectif Sauvons la recherche, pourrait être opposée à l'idéologie libérale qui se déploie dans le monde académique ».

Loin de nous l'idée de vouloir devenir la passionaria du futur mouvement universitaire (au risque de désespérer davantage Éric Dacheux), ou de nous prendre pour l'avant-garde éclairée de ce qui serait devenu le prolétariat académique. II n'en reste pas moins que notre texte est un équilibre assumé entre rigueur analytique et un ton parfois plus engagé défendant une certaine idéalisation, ce que nous avons synthétisé en revendiquant le statut de « pamphlet académique ». 
À cet égard, dans l'article d'Éric Martin, Gilles Labelle et Maxime Ouellet (2013), nous trouvons de précieuses ressources. À partir de leur exercice ô combien stimulant de cadrage conceptuel sur l'Université comme « institution », ils décrivent une institution comme un mixte entre un monde commun visible, objectivé, et une forme de subjectivité liée aux êtres humains qui composent ce collectif institutionnalisé.

\begin{abstract}
« L'institution universitaire, comme toutes les autres institutions, repose sur ce binôme : d'un côté, un commun mis en partage (même incomplètement), un monde commun, qui se rend visible dans des formes qui ont une consistance objective, dans des règles, dans des normes, dans des codes (qui supposent, forcément, des possibilités de sanctions) et, d'un autre côté, un type humain, une forme de subjectivité arrimée à ces formes, baignant dans ce commun, et lui correspondant en quelque façon » (Martin, Labelle, Ouellet, 2013 : 254).
\end{abstract}

Plus loin, ils répondent (sans le savoir) à Julien Duval et Julie Bouchard, en mettant en évidence le fait que les divergences et conflits internes aux universitaires ne leur interdisent toutefois pas de trouver quelques éléments formant point commun, comme nous aspirions à le montrer :

« Ces différences quant au sens à donner aux "missions" de l'Université n'ont cependant nullement empêché que soit forgée dans l'histoire occidentale la représentation d'un lieu, précisément désigné comme "institution universitaire", occupant une place distincte dans le tissu institutionnel des sociétés en ce qu'il était censé : a) entretenir les conditions d'une transmission du "haut savoir" à ceux qui n'y avaient pas eu accès jusque-là, de même que b) générer le développement d'un climat favorable aux efforts visant à l'avancement et au "progrès" des connaissances » (ibid.).

Sur ce plan de l'idéalisation du modèle universitaire, remercions Éric George (2013 : 246) de nous avoir fait connaître (dans sa conclusion) cette remarquable citation de Cornelius Castoriadis $^{10}$, qui dépeint mieux que nous n'aurions su l'écrire, l'idéal pédagogique pour l'Université auquel nous aspirons : « Une éducation pour l'autonomie et vers l'autonomie, qui amène ceux qui sont éduqués à s'interroger constamment pour savoir s'ils agissent en connaissance de cause plutôt qu'emportés par une passion ou par un préjugé »».

Pour conclure sur ce point, Gilles Labelle, Éric Martin et Maxime Ouellet (2013:252) nous apostrophent en introduction : « [L'auteur] nous paraît parfois hésiter entre une analyse évoquant une transformation, en cours présentement, qui changerait l'Université en "autre chose" qu'elle-même et un autre diagnostic évoquant plutôt une sorte de dérapage de l'institution universitaire ». Si des lecteurs aussi attentifs et connaisseurs du sujet ont vu une ambiguïté dans notre position, c'est que nous n'avons pas été assez clair. Alors disons plus explicitement que l'Université subit une somme de transformations, d'inflexions organisationnelles, de révisions de ses priorités, qui vont au-delà de simples dérapages, mais qui remettent en cause un modèle ancestral et qui oblige les universitaires à réagir avant qu'il ne soit trop tard, à reprendre en mains leur destin professionnel, en commençant par faire un effort de lucidité sur le diagnostic, auquel de nombreux travaux déjà parus

\footnotetext{
10 Accès : http://www.monde-diplomatique.fr/ I998/02/CASTORIADIS/ I 0046.html. Consulté le 26/08/I 3.
} 
ou des réflexions en cours contribuent, comme nous l'avons indiqué dans notre précédent article (Mercier, 20 I 2). Puisque plusieurs contributeurs de cette rubrique ont souligné que notre partie conclusive sur les pistes à explorer pour sortir de l'ornière était un peu expéditive, nous profiterons de notre droit de réponse, pour exposer sur un mode plus personnel des éléments programmatiques en quelque sorte. Mais commençons par dire notre relatif désaccord, après une utilisation féconde de la notion d'espace public appliquée aux universités, avec le diagnostic final très sévère dressé par Éric Dacheux (2013 : 206) : « En vérité, si la loi LRU et le new public management on fait si rapidement autant de dégâts, c'est peutêtre parce qu'ils ont été, dans les faits, peu combattus et que, depuis longtemps, beaucoup d'universitaires ont renoncé à être des chercheurs libres, préférant s'abriter derrière les dysfonctionnements du système plutôt que de lutter pour le réformer 》. Ou encore : « Nous sommes victimes parce que nous n'avons pas eu le courage de nous engager corps et âme dans la lutte ; nous avons perdu la bataille parce que nous avons refusé de mener la guerre » (ibid. : 207). Des mobilisations ont eu lieu à plusieurs reprises dans la communauté universitaire, y compris contre la $L R U$, mais dans une ampleur insuffisante pour faire bouger les lignes, c'est juste.

Récemment encore, en quelques jours, une grande partie de la communauté a su se mobiliser dans l'urgence face à la suppression en catimini, à rebours des engagements pris par le gouvernement, de la procédure de qualification des enseignants-chercheurs au cNu. Cette décision fut votée le 2 I juin 2013 au Sénat, à la sauvette, par une poignée de sénateurs, les autres écrasant les universitaires de leur mépris et de leur indifférence, par leur absence massive dans l'hémicycle pour voter la réforme de l'enseignement supérieur. Un groupe de sénateurs verts irresponsables jugea utile de déposer un amendement venant bousculer l'équilibre des recrutements académiques sans aucune solution de rechange. Et le professeur Vincent Berger, rapporteur des Assises de l'enseignement supérieur et de la recherche et président de l'université Paris Diderot, défendit, aussi surpris que ravi, ce vote dans une interview à l'Agence éducation et formation (AEF - 25/06//3). II y déploya une rhétorique qui relèva de la plus pure sophistique. II se déclara très favorable à la suppression de ce sas de contrôle de la qualification par le cNu, pour trier entre les thèses de complaisance et les bonnes, en faisant semblant de croire que toutes les thèses se valent. Depuis quand croit-il que tous les diplômes se valent, que les masters de toutes les universités, de France et d'ailleurs, se valent? Depuis quand croit-il que toutes les revues se valent? Qu'il suffirait d'avoir publié un article scientifique pour qu'il ait la même valeur?

Non, les plus de 15000 membres de la communauté universitaire qui ont signé en trois jours la pétition lancée par la commission permanente du Conseil national des universités (CP-CNU) « n'avouent pas piteusement », comme Vincent Berger le prétendait avec une ficelle rhétorique, que tous les doctorats ne se valent pas. Au contraire, c'est une réalité que nous ne cessons de rendre publique pour mieux la combattre. Et si la communauté se bat pour une meilleure reconnaissance professionnelle du doctorat, c'est pour les bonnes thèses! Dans cet entretien, le professeur Berger est bien obligé d'admettre qu'il peut y avoir un risque 
« d'endorecrutement » au niveau local avec la suppression du filtrage national par le cNu.Tous les communiqués d'associations universitaires (syndicales, disciplinaires, de droite comme de gauche) l'ont dénoncé comme un risque évident de « népotisme » et de clientélisme, tel qu'il exista naguère, avant l'instauration de la procédure de qualification, et qui arrive toutefois à persister encore parfois. Obligé d'admettre l'existence de ce risque, il plaide pour une « évaluation des universités » sur ce point. Autrement dit, chaud partisan de l'autonomie il voudrait instaurer un contrôle de plus. Nouvelle incohérence de cette piètre argumentation.

Vincent Berger reprend aussi à son compte l'argument absurde du « temps perdu », « chronophage » disait la sénatrice verte à la tribune. Mais, les comités de sélection locaux doivent trier entre les dossiers de candidature reçus en faisant deux rapports à chaque fois. Le non-filtrage a priori des candidats conduirait des vagues de candidats sur chaque poste, que chaque collègue, chaque université mettrait des heures à traiter dans les services Ressources humaines, à trier, à rapporter, à contacter pour refus d'audition, à payer pour le retour des dossiers envoyés. Supprimer la qualification ce n'est pas libérer du temps de travail pour les universitaires, c'est transférer sur chaque université et chaque comité de sélection ce temps de travail de filtrage réalisé par une poignée de collègues élus ou nommés, mais volontaires, qui acceptent de sacrifier une partie de leur temps de recherche au cNu, pour le bien commun de chaque discipline.

Quant à l'idée que cette proposition de suppression était issue des Assises de l'enseignement supérieur, la vérité est que c'était surtout le fruit d'une manipulation des débats de ces Assises. Ces procédures consultatives pseudo-démocratiques sont un piège grossier et désormais bien connu, grâce à de nombreux travaux universitaires (Blatrix, Blondiaux, Fourniau, 2010 ; Monnoyer-Smith, 201 I). On réunit des acteurs concernés en leur faisant miroiter la lune en plein jour : « Exprimezvous », « dites-nous ce que vous avez sur le cœur 》, « soyez force de proposition ». À la fin, il suffit que quelques personnes aient exprimé une proposition qui est celle que le législateur et le gouvernement voulaient entendre pour qu'elle soit retenue et présentée fallacieusement comme issue de la concertation, alors même (comme c'est le cas pour l'abolition de la qualification) qu'une majorité des collègues et des présents aux Assises étaient contre dès le début.

Devant l'immédiate et instinctive mobilisation, les trois sénateurs écologistes, apprentis fossoyeurs de l'Université, ont « piteusement »-comme dirait Vincent Berger - rétropédalé dans un communiqué de presse : « Nous pouvons vous rassurer, cette mesure tombera en Commission mixte paritaire mercredi », et une autre sénatrice verte, Esther Benbassa, soutenant a priori ce projet funeste a, dans un courriel rendu public, répondu à plusieurs personnes qui l'avait interpelée sur sa messagerie : « La suppression pure et simple, sans contrepartie, de la procédure de qualification était une erreur ».

Parmi les milliers de signataires de la pétition certains défendent la qualification parce qu'ils y croient dur comme fer, d'autres parce qu'ils pensent que si le cNu a des défauts, il en a moins que le retour à la situation ex ante. Mais, tous sont 
convaincus aussi et surtout que l'intrusion de politiciens ignorants des logiques profondes de l'Université dans nos affaires, pour se livrer à un travail de sape de l'Université à l'œuvre depuis plus de dix ans maintenant, par « réformes » successives, est devenue insupportable, que trop c'est trop. Pour autant, la prise de position de Vincent Berger donne pleinement raison à Éric Dacheux ou Julien Duval qui insistent sur le fait que les agents extérieurs bénéficient de la collaboration de collègues sur qui s'appuyer pour entretenir leurs noirs desseins. Des dispositifs qui font relatif consensus (même si les critiques sur le cNU alimentent de nombreux débats en interne) peuvent donc réveiller une réflexion de mobilisation qu'Éric Dacheux décrit un peu trop comme moribonde.

À titre plus personnel, notre forme d'engagement passe par l'acceptation de postes en responsabilité pour tenter, ensuite, d'y faire fructifier des principes auxquels nous croyons et qui offrent une résistance aux tendances lourdes (hélas, le succès n'est pas au rendez-vous sur tous les points). Cela implique que nous ne plaidons pas pour une radicalité, avec défense ardente d'un passé révolu, fondée sur l'idée que tout était mieux avant et que toute évolution est néfaste en soi, donc impossible. Nous épousons plutôt la posture réformiste défendue par Marc Lits et Évelyne Léonard (2013 : 272-273). Oui, « des questions se posent sur la structure et l'organisation hiérarchique. II faut clarifier le choix entre centralisation versus décentralisation et les enjeux d'une réelle subsidiarité (laisser la décision à l'endroit où elle peut être prise de manière efficace et économe) qui définit clairement les lieux de décision ». Oui, « il faut définir des priorités plutôt que démultiplier les attentes sur tout et partout au prix de l'augmentation de la charge de travail ». Oui, il nous faut savoir conduire « une réflexion sur les nouveaux rapports qui se construisent face aux étudiants, aussi bien dans les structures de décision que dans les méthodes d'enseignement ». À chaque fois, l'enjeu est de définir la place des personnels qui font vivre l'Université, qui lui donnent corps, dans le processus de décision.

Par exemple, l'idéal de subsidiarité nous semble un principe intangible. La course au gigantisme, la présidentialisation de la gouvernance universitaire sont autant de «bons prétextes » à une centralisation bureaucratique du processus de décision, aspirant des périphéries vers un centre boursoufflé, des forces vives administratives dont l'absence se fait cruellement et rapidement sentir au plus près du terrain. Nous défendons également un idéal de société de confiance. Qu'il nous soit permis de nous citer: « Les modalités participatives internes sont sans aucun doute à repenser pour les revivifier. II faut sortir d'une société de la défiance, où le principe de contrôle comptable bureaucratisé a priori l'emporte. II faut débrider l'esprit d'initiative, en instaurant un climat de confiance a priori dans la gestion des fonds alloués ou obtenus par contrats extérieurs, avec un système strict de sanction a posteriori si des abus étaient découverts 》 (Mercier, 2012 : 230). À travers cette proposition qui peut sembler anodine, c'est bien d'un bouleversement qu'il s'agit. Cela voudrait dire pouvoir dépenser librement l'argent dont on dispose, cela voudrait dire pouvoir acheter en ligne au meilleur 
tarif, plutôt que de passer par des prestataires de service détenteurs d'un « marché public » et qui ont, dès lors, un monopole sur certains types d'achat et nous empêchent de saisir d'intéressantes opportunités, d'acheter le matériel que l'on souhaite, le plus adapté. Cela signifie rompre avec un contrôle budgétaire tatillon, rompre avec le transfert du pouvoir de décider vers d'autres agents que les premiers concernés.

II convient aussi d'entendre les aspirations à une certaine professionnalisation des cursus. Nous y avons nous-mêmes contribué, en portant un projet de création d'une licence professionnelle sur le journalisme numérique, qui reste assez peu fréquent dans le secteur des sciences humaines et sociales. Mais tout ceci peut et doit se faire dans le respect des missions traditionnelles de l'Université. Autorisons-nous une anecdote personnelle à ce sujet. En qualité de directeur d'une Unité de formation et de recherche (UFR), nous avons participé à la cellule de réflexion interne à notre université pour définir les critères dits de performance. Ce fut l'occasion d'un dialogue de sourd stupéfiant, sur deux thématiques que nous avions mises en avant. Alors qu'un air d'évidence accompagnait la proposition de notre président de mettre en place les outils pour mesurer le taux d'insertion professionnelle de nos diplômés, nous demandions comment nous allions mesurer notre «performance » sur la transmission des savoirs (mission multiséculaire des universités!). En réponse un rire étouffé ou nerveux traversa la petite assemblée, comme si nous venions d'énoncer une incongruité. M'insurgeant du fait que l'on mettait en exergue uniquement une des nouvelles missions de l'Université sans valoriser ce qui faisait notre marque de fabrique depuis toujours, j'obtins enfin une réponse courtoise mais ferme : « Non mais ça, c'est une évidence ! ». Fermez le ban! L'autre sujet concernait la montée du stress au travail, ce que les psychologues nomment la charge mentale. Je défendis l'idée de créer un indicateur de bien-être au travail. Les rires se transformèrent en grimaces, avant d'entendre une incroyable négation du problème, alors même que la somme des transformations subies contribue à une évidente dégradation des conditions de travail de tous les personnels. Redisons-le, chacun doit pouvoir, face à toute situation de travail intolérable engendrée par les nouveaux préceptes du management, réclamer de voir s'appliquer non pas la gestion des ressources humaines, mais bien une gestion humaine des ressources.

\section{Conclusion}

Au-delà de quelques amalgames et d'un excès de pessimisme, Éric Dacheux (2013) a le mérite de rappeler chacun à sa responsabilité quant au fait de subir ou non notre sort professionnel. De manières diverses, des voies existent pour lutter contre un mouvement que certains présentent comme inexorable et infiniment désirable et pour faire entendre notre voix. On peut le résumer par un triptyque : chercher, faire savoir, s'engager. 


\section{Chercher}

Comme a tenté de le faire ici Questions de communication, comme le font des collègues en organisant des séminaires, des colloques, des journées d'étude, soyons porteurs d'une démarche autoréflexive. Ne perdons pas de vue collectivement l'obligation qui nous incombe de réfléchir aux enjeux sociopolitiques du système universitaire et aux transformations en devenir qui vont encore bouleverser nos équilibres professionnels. On pense notamment aux MOOC (massive open online course) et à leurs effets induits, évoqués rapidement par Marc Lits et Évelyne Léonard (2013). Les sciences de l'information et de la communication (sıc) sont particulièrement bien outillées conceptuellement pour s'emparer de la question, grâce aux travaux réalisés sur les technologies de l'information et de la communication appliquées à l'éducation (TICE). Dans les appels à financement, proposons des projets de recherche sur l'Université et ses transformations. Non par nombrilisme ou par apparente facilité d'affinité entre le sujet et notre vécu, mais parce que la construction de notre cause dépend aussi de la rigueur de notre discours critique. Et puisque nous sommes aussi dans les comités de financement, intégrons cette exigence éthique et politique, en regardant avec bienveillance de tels projets et défendons des travaux sur les universités dans ces commissions.

\section{Faire savoir}

Profitons de chaque tribune sur l'extérieur qui nous est donnée (médias, réunions publiques, dialogues avec des citoyens, etc.) pour expliciter la dégradation des conditions de travail subies et pour dénoncer les mensonges et idées fausses prononcées à notre égard. Sans forcer sur le misérabilisme, en nous faisant indûment passer pour des mineurs risquant notre vie au fond de puits dangereux, il est permis d'affirmer haut et fort que la condition des personnels universitaires s'est suffisamment dégradée pour en faire parfois un métier pénible, à l'instar de ce que peuvent vivre désormais les personnels hospitaliers. Tant que cette vérité n'aura pas acquis un caractère d'évidence partagée aux yeux de nos interlocuteurs (élus, bailleurs de fonds, citoyens, étudiants, journalistes...), nous serons en butte à des discours nous présentant comme des privilégiés qui devraient déjà être bien contents du sort qui est le leur, ou pire, qui peuvent bien accepter encore quelques sacrifices, des remises en cause, des tâches en plus, au vu de leurs « privilèges ».

\section{S'engager}

Bien sûr, la tentation du repli sur soi, dans l'individualisme de nos pratiques, loin de l'espace public académique, est grande. Bien sûr, certains collègues la pratiquent déjà (Éric Dacheux le souligne hélas avec raison). Bien sûr, les schémas simplistes hantent les têtes de nombreux politiciens sur une institution qu'ils ne connaissent 
pas au mieux, qu'ils méprisent au pire (eux qui ont soigneusement évité l'Université durant leurs cursus) et cela finit par être décourageant. Bien sûr, on peut se replier sur une position de principe visant à ne pas vouloir se prêter au jeu des institutions présidentialisées et centralisées. Mais, si tous ceux qui croient à d'autres idéaux pour l'Université se détournent systématiquement des lieux de débat et de décision, alors nous laissons le néomanagérialisme s'imposer sans combattre. Ne fuyons pas trop les lieux de décision dès lors qu'il y reste quand même un peu de marges d'autonomie pour atténuer les effets néfastes, voire pour réussir à pervertir de l'intérieur les logiques politiques à l'œuvre, en profitant de ces lieux de décision (conseils divers...) pour instiller dans le système des principes ou objectifs contraires à l'air du temps. Et si nous-même avons un peu levé le pied après avoir beaucoup donné au collectif (Aeres, direction d'une UFR, tête de liste à l'élection à la présidence de notre université...), nul doute qu'il s'agit d'un cycle logique d'alternance pendant que d'autres collègues prennent leur part de responsabilité. Le combat se poursuit. Il est intellectuel (chercher), communicationnel (faire savoir) et politique et organisationnel (s'engager).

\section{Références}

Ansart P., 1983, La Gestion des passions politiques, Lausanne, Éd. L’Âge d'homme.

Blatrix C., Blondiaux L., Fourniau J.-M., 20 I0, Le débat public : une expérience française de démocratie participative, Paris, Éd. La Découverte.

Bouchard J., 2013, « Les classements d'établissements d'enseignement supérieur et de recherche : des miroirs déformants aux instruments de régulation », Questions de communication, 23, pp. 175-196.

Braud P., 1996, L'Émotion en politique, Paris, Presses de Sciences Po.

Dacheux É., 2013, «Pas de démocratie universitaire sans courage des universitaires », Questions de communication, 23, pp. 197-210.

Duval J., 20 I3, «Retour sur l'évolution universitaire en France », Questions de communication, 23, pp. $211-230$.

George É., 2013, «La dérive des universités, vue de l'autre côté de l'océan Atlantique », Questions de communication, 23, pp. 231-250.

Gillot D., Dupont A., 2013, Rapport d'information fait au nom de la commission sénatoriale pour le contrôle de l'application des lois sur la mise en œuvre de la loi n² 2007-1 199 du 10 août 2007 relative aux libertés et responsabilités des universités, par $M^{\text {me }}$ Dominique Gillot et M. Ambroise Dupont, sénateurs, Paris, Sénat. Accès : http://www.senat.fr/rap/rl 2-446/ r|2-446|.pdf. Consulté le 13// ///3.

Girardet R., 1986, Mythes et mythologies politiques, Paris, Éd. Le Seuil.

Hobsbawm E., RangerT., 1983, The Invention of Tradition, Cambridge, Cambridge University Press.

Johnson A., Van Ostern T., White A., 2012, The Student Debt Crisis, Washington, Center for American Progress, 25 oct. Accès: http://www.americanprogress.org/wp-content/ uploads/2012/10/WhiteStudentDebt-4.pdf. Consulté le 23/08/13. 
Labelle G., Martin E., Ouellet M., 2013, «"Dérapage" ou remise en cause de l'institution universitaire », Questions de communication, 23, pp. 25I-260.

Lee D., 20 I3, Household Debt and Credit: Student Debt, New York, Federal Reserve Bank of NewYork, 28 febr.Accès: http://www.newyorkfed.org/newsevents/mediaadvisory/20 I3/ Lee0228 I 3.pdf. Consulté le 28/08// 3.

Lits M., Léonard E., 2013, « Les universités belges entre concurrence et excellence », Questions de communication, 23, pp. 261-274.

Mercier A., 2012, «Dérives des universités, périls des universitaires 》Questions de communication, 22, pp. 197-234.

Monnoyer-Smith L., 20 I I, Communication et délibération. Enjeux technologiques et mutations citoyennes, Paris, Lavoisier/Hermès.

Musselin C., 1997, « Les universités sont-elles des anarchies organisées? 》, pp. 29I-308, in : Chevallier J., dir., Désordre(s), Paris, Presses universitaires de France.

Nguyen M., 2012, Degreeless in Debt:What happens to Borrowers Who Drop out,Washington, Education Sector, febr. Accès : http://wnw.educationsector.org/sites/default/files/publications/ DegreelessDebt_CYCT_RELEASE.pdf. Consulté le 25/08/I3.

Ricœur P., 1997, L'idéologie et l'utopie, Paris, Éd. Le Seuil.

The Institute for College Access \& Success, 20 I2, Student Debt and the Class of 201 I, Oakland/ Washington, The Institute for College Access \& Success/The Project on Student Debt, oct. Accès : http://projectonstudentdebt.org/files/pub/classof20 I I.pdf. Consulté le 28/08/I3. 